\title{
Synthesis and Free Radical-scavenging Activities of Di-mannich Bases of Cyclovalone Derivatives
}

\author{
HAYUN HAYUN*, CATUR JATMIKA, EUIS MARAS PURWATI, SANDI SALIM, \\ ROSITA KURNIAWAN, ELIZABETH GREFFIANA CHANDRA, ADAM ARDITYA FAJRIAWAN \\ and AULIKA DESTHAHRINA NARESWARA
}

Faculty of Pharmacy, Universitas Indonesia, Depok, West Java,16424, Indonesia.

*Corresponding author Email: hayun.ms@ui.ac.id

http://dx.doi.org/10.13005/ojc/330607

(Received: July 30, 2017; Accepted: September 01, 2017)

\begin{abstract}
Novel di-Mannich bases of cyclovalone derivatives (1) have been synthesized and evaluated their antioxidant activity using DPPH free radical-scavenger method. The structures of the compounds were confirmed on the basis of FT-IR, ${ }^{1} \mathrm{H}-\mathrm{NMR},{ }^{13} \mathrm{C}-\mathrm{NMR}$ and mass spectral data. The result of antioxidant evaluation showed that di-Mannich derivative of cyclovalone with diethylamine ((2E,6E)-2,6-bis(\{3-[(diethylamino)methyl]-4-hydroxy-5-methoxyphenyl $\}$ methylidene) cyclohexan-1-one) (2a) exhibited the highest antioxidant activity with $\mathrm{IC}_{50}=39.0 \mu \mathrm{M}$. Structure-activity relationship study showed that the higher pKa of the Mannich base, the higher activity (the lower $\mathrm{IC}_{50}$ ) of the compound.
\end{abstract}

Keywords: Cyclovalone, Mannich base, Synthesis, Antioxidant, Radical scavenger.

\section{INTRODUCTION}

Oxidative stress, generating by an imbalance between free radical production and antioxidant defenses, is associated with the damage of various species of molecules including proteins, lipids, and nucleic acids. A role for oxidative stress has been postulated to contribute significantly to the pathogenesis of atherosclerosis, inflammatory conditions, certain cancers, and the process of aging. Besides the complex system of antioxidant metabolites and enzymes that naturally prevent cell damage, the exogenous antioxidant may sometimes be required to keep reactive oxygen species at optimum level ${ }^{1-3}$.

Cyclovalone, (2E,6E)-2,6-bis[(4-hydroxy3-methoxyphenyl)methylidene]cyclohexan-1-one (1) is a curcumin mono-carbonyl analog in which the pentane-2,4-dione chain of the curcumin is replaced by a cyclohexanone ring. Cyclovalone demonstrated antioxidant activity, antitumor, anti inflammatory, hepatoprotective and cytotoxic activity $^{4-7}$. The structure-activity relationships (SAR) including the antioxidant activity of cyclovalone derivatives have been studied. However, there has 
been no report about the effect of aminoalkyl substituents ${ }^{5,8}$

A Mannich reaction is a suitable method to introduce aminoalkyl group into a molecule. In several instances, the Mannich derivatives exhibit better biological activity than the corresponding parent analogs. Moreover, the presence of Mannich side chain increases the solubility and hence the bioavailability of the compounds ${ }^{2,9-13}$. Herein we report the synthesis and antioxidant activity of Mannich bases derivatives of cyclovalone.

\section{MATERIALS AND METHODS}

\section{Chemistry}

All used chemicals were purchased from Merck or Aldrich Company and used without further purification. Thin layer chromatography (TLC) was carried out on silica gel $60 \mathrm{~F}_{254}$ plates (Merck) and spots were detected under an ultraviolet and visible light. Melting points were determined in the capillary tube using electrothermal digital melting point apparatus (Stuart Scientific). The infrared spectra were recorded with FTIR 8400S Spectrometer (Shimadzu). NMR spectra were recorded on NMR spectrometer (Agilent) at $500 \mathrm{MHz}$ for ${ }^{1} \mathrm{H}$ and 125 $\mathrm{MHz}$ for ${ }^{13} \mathrm{C}$ using TMS as an internal standard. Highresolution mass spectra (HRMS) were measured with a Waters LCT Premier XE (ESI-TOF) system in positive or negative mode. The absorption in the determination of free radical-scavenging activity was measured using UV-Vis Spectrophotometer 1601 (Shimadzu).

\section{General synthesis of di-Manncih bases of cyclovalone derivatives (2a-e)}

The di-Mannich bases of cyclovalone were synthesized by Mannich reaction of compound 1 according to the method of synthesis of di-Mannich bases of 1,5-bis(4-hydroxy-3-methoxyphenyl) penta-1,4-dien-3-one reported previously ${ }^{7}$, with little modification: The solution of $2 \mathrm{mmol}$ of compound 1 in acetonitrile $(50 \mathrm{ml})$ was added to mixture of $16 \mathrm{mmol}$ paraformaldehyde and $16 \mathrm{mmol}$ of corresponding secondary amine in acetonitrile $(50 \mathrm{ml})$ which previously was heated at $80^{\circ} \mathrm{C}$ for 10 minutes. The reaction mixture then was refluxed until the disappearance of 1 . The completion of the reaction was monitored by TLC for $5-27 \mathrm{~h}$. The reaction solvent was removed in vacuo using a rotary evaporator, the crude products were washed with cold acetonitrile and then purified by recrystallization or column chromatography to give compound 2a-e.

(2E,6E)-2,6-bis(\{3-[(diethylamino)methyl]-4hydroxy-5-methoxyphenyl\}methylidene) cyclohexan-1-one (2a):

The crude product was purified by recrystallization from ethylacetate-hexane (1:25) gave a yellowish orange crystalline powder at $84.3 \%$ yield, m.p. $=58-60^{\circ} \mathrm{C} . \mathrm{IR}(\mathrm{KBr}), v_{\max }, \mathrm{cm}^{-1}$ : 2972, 2935, 2833, 1639, 1589, 1246 1087, and 1039. ${ }^{1} \mathrm{H}-\mathrm{NMR}\left(500 \mathrm{MHz}, \mathrm{CDCl}_{3}\right.$ ) $\delta / \mathrm{ppm}: 7.71$ $(2 \mathrm{H}, \mathrm{s}, \mathrm{C}=\underline{\mathrm{CH}}-), 6.97\left(2 \mathrm{H}, \mathrm{s}, \mathrm{H}_{\mathrm{Ar}}\right), 6.80\left(2 \mathrm{H}, \mathrm{s}, \mathrm{H}_{\mathrm{Ar}}\right)$, $3.91\left(6 \mathrm{H}, \mathrm{s}, \mathrm{OCH}_{3}\right), 3.82\left(4 \mathrm{H}, \mathrm{s}, \mathrm{Ar}-\mathrm{CH}_{2}-\mathrm{N}\right.$ - $\left._{\text {diethylamine }}\right)$, $2.94\left(4 \mathrm{H}, \mathrm{t},=\mathrm{C}-\mathrm{CH}_{2}-\mathrm{C}_{\text {cyclohexanone }}\right), 2.66\left(8 \mathrm{H}, \mathrm{q},-\mathrm{CH}_{3}-\right.$ $\left.\underline{\mathrm{CH}}_{2}-\mathrm{N}_{\text {diethylamine }}\right), 1.83\left(2 \mathrm{H}, \mathrm{p}, \mathrm{C}-\underline{\mathrm{CH}}_{2}-\mathrm{C}_{\text {cyclohexanone }}\right)$ and $1.14\left(12 \mathrm{H}, \mathrm{t}, \mathrm{CH}_{2}-\mathrm{CH}_{3 \text { diethylamine }}\right) \cdot{ }^{13} \mathrm{C}-\mathrm{NMR}(125 \mathrm{MHz}$,

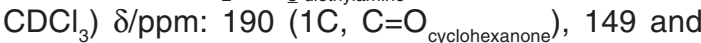
147 (4C, $\left.\mathrm{C}_{\text {Ar-O }}\right), 133$ and 137 (4C, -C=C-), 126, 123, 121 , and $113\left(8 \mathrm{C}, \mathrm{C}_{\mathrm{Ar}-\mathrm{H}}\right), 57\left(2 \mathrm{C}\right.$, Ar- $\left.\underline{\mathrm{C}}-\mathrm{N}_{\text {diethylamine }}\right)$, $56\left(2 \mathrm{C}, \mathrm{Ar}-\mathrm{O}_{\mathrm{C}} \mathrm{H}_{3}\right), 46\left(4 \mathrm{C},-\mathrm{N}-\underline{\mathrm{CH}}_{2 \text { diethylamine }}\right), 28$ (2C, $\left.=\mathrm{C}-\underline{\mathrm{C}} \mathrm{H}_{2}-\mathrm{C}_{\text {cyclohexanone }}\right), 23\left(1 \mathrm{C}, \mathrm{C}-\underline{\mathrm{C}} \mathrm{H}_{2}-\mathrm{C}_{\text {cyclohexanone }}\right)$, and $11\left(\mathrm{C}-\mathrm{CH}_{3}\right)$. HRESIMS (m/z): found 537.3315 $\left([\mathrm{M}+\mathrm{H}]^{+}\right)$, calculated masses of $\mathrm{C}_{32} \mathrm{H}_{45} \mathrm{~N}_{2} \mathrm{O}_{5}$ : 537.3328 (error - 2.4 ppm).

(2E,6E)-2,6-bis(\{3-[(dimethylamino)methyl]-4hydroxy-5-methoxyphenyl\}methylidene) cyclohexan-1-one (2b)

The crude product was purified by column chromatography on silica with mixture of chloroform, methanol and ethanol (10:1:1) as mobile phase gave a brown crystalline powder at $44.3 \%$ yield, m.p. $=182-184^{\circ} \mathrm{C} . \mathrm{IR}(\mathrm{KBr}), v_{\max }, \mathrm{cm}^{-1}: 2993,2829$, 1605, 1558, 1488, 1250 1086, and 1008. ${ }^{1} \mathrm{H}-\mathrm{NMR}$

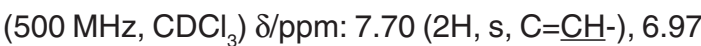
$\left(2 \mathrm{H}, \mathrm{s}, \mathrm{H}_{\mathrm{Ar}}\right), 6.79\left(2 \mathrm{H}, \mathrm{s}, \mathrm{H}_{\mathrm{Ar}}\right), 3.89\left(6 \mathrm{H}, \mathrm{s}, \mathrm{OCH}_{3}\right)$, $3.68\left(4 \mathrm{H}, \mathrm{s}, \mathrm{Ar}-\mathrm{CH}_{2}-\mathrm{N}_{\text {dimethylamine }}\right), 2.93(4 \mathrm{H}, \mathrm{t}$, =C$\left.\mathrm{CH}_{2}-\mathrm{C}_{\text {cyclohexanone }}\right), 2.35\left(12 \mathrm{H}, \mathrm{s}, \mathrm{CH}_{3}-\mathrm{N}-_{\text {dimethylamine }}\right)$, and $1.82\left(2 \mathrm{H}, \mathrm{p}, \mathrm{C}-\mathrm{CH}_{2}-\mathrm{C}_{\text {cyclohexanone }}\right) .{ }^{13} \mathrm{C}-\mathrm{NMR}(125$ $\left.\mathrm{MHz}, \mathrm{CDCl}_{3}\right) \delta / p p m: 190\left(1 \mathrm{C}, \mathrm{C}=\mathrm{O}_{\text {cyclohexanone }}\right), 149$ and $148\left(4 \mathrm{C}, \mathrm{C}_{\mathrm{Ar}-\mathrm{O}}\right), 134$ and $137(4 \mathrm{C},-\mathrm{C}=\mathrm{C}-), 127$, 124,122 , and $114\left(8 \mathrm{C}, \mathrm{C}_{\mathrm{Ar}}\right), 63$ (2C, Ar- $\underline{\mathrm{C}}-$ $\left.\mathrm{N}_{\text {dimethylamine }}\right), 56\left(2 \mathrm{C}, \mathrm{Ar}-\mathrm{O}_{\mathrm{C}} \mathrm{H}_{3}\right), 45\left(4 \mathrm{C},-\mathrm{N}-\mathrm{CH}_{3}\right.$ dimethylamine $), 29\left(2 \mathrm{C},=\mathrm{C}-\underline{\mathrm{CH}}_{2}-\mathrm{C}_{\text {cyclohexanone }}\right)$, and $23(1 \mathrm{C}$, $\left.\mathrm{C}-\mathrm{CH}_{2}-\mathrm{C}_{\text {cyclohexanone }}\right)$. HRESIMS $(\mathrm{m} / \mathrm{z})$ : found $479.2539\left([\mathrm{M}-\mathrm{H}]^{-}\right)$, calculated masses of $\mathrm{C}_{28} \mathrm{H}_{35} \mathrm{~N}_{2} \mathrm{O}_{5}: 479.2546$ (error -1.5 ppm). 


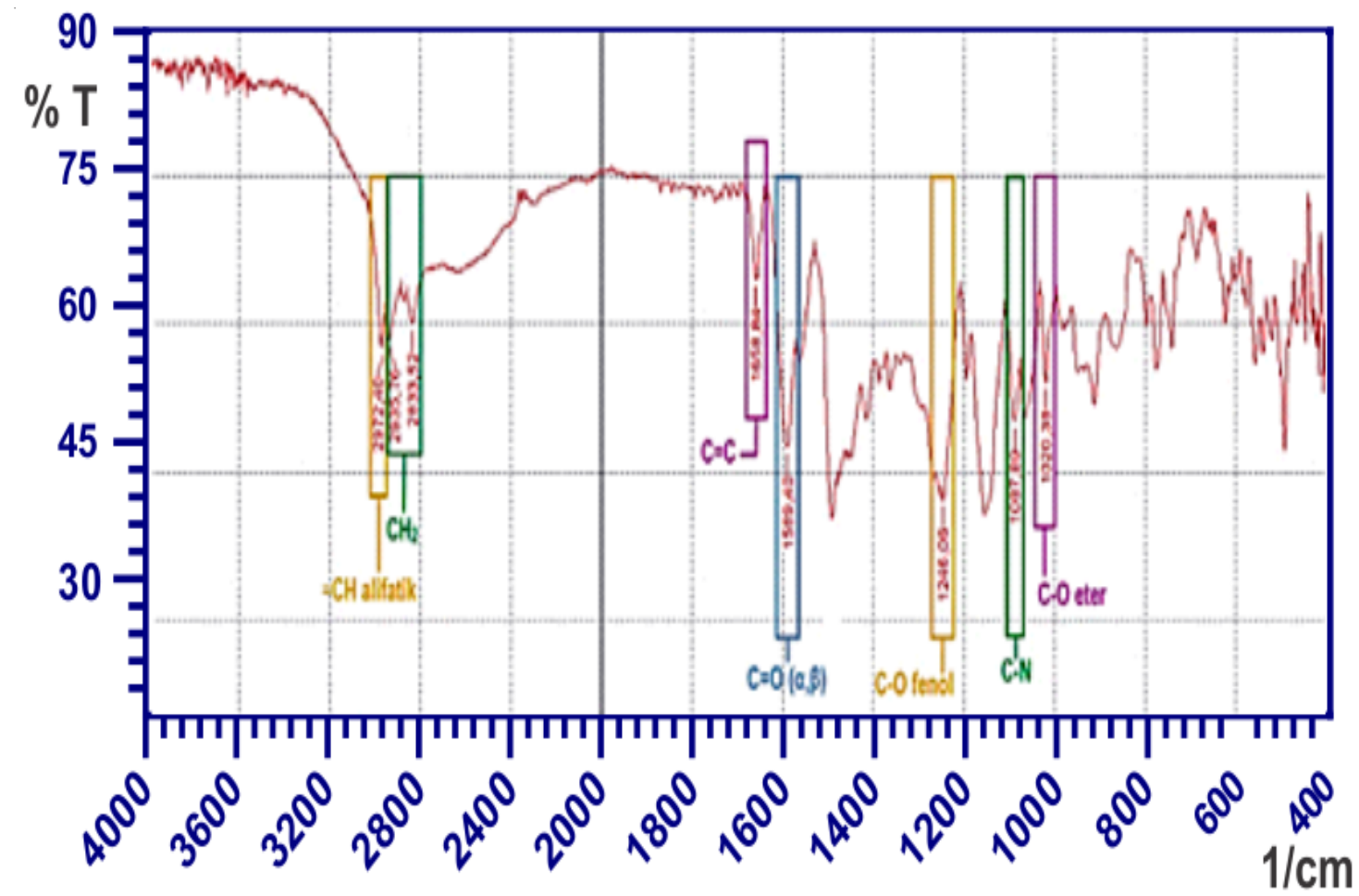

IR Spectrum of compound $2 \mathrm{a}$ in $\mathrm{KBr}$

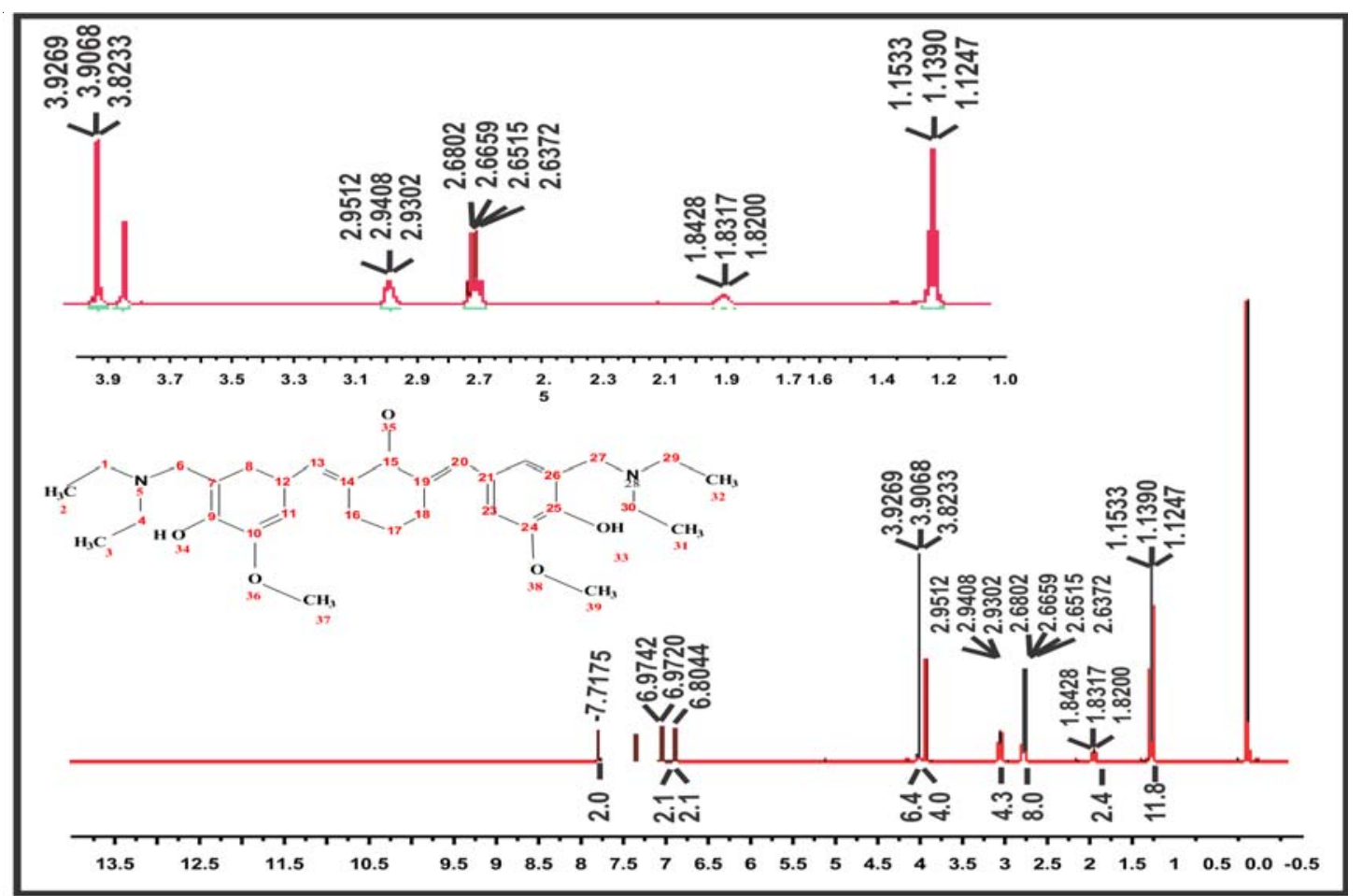

${ }^{1} \mathrm{H}$ NMR Spectrum of compound $2 \mathrm{a}$ 


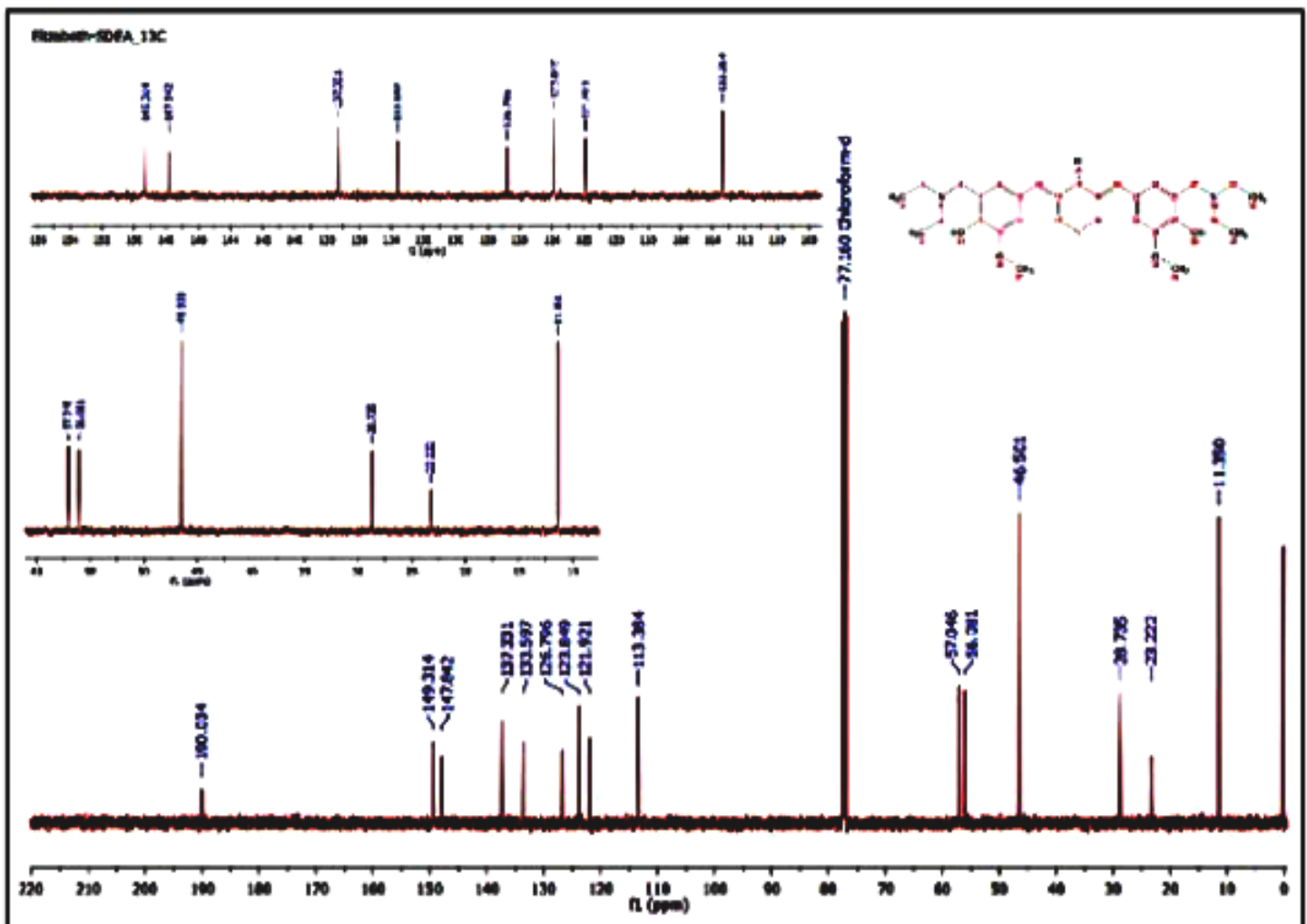

${ }^{13} \mathrm{C}$ NMR Spectrum of compound $2 \mathrm{a}$

Tous 15

2510N

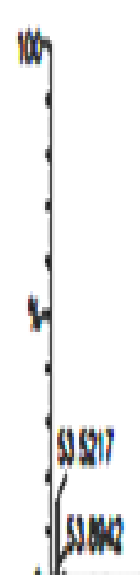

\section{W.IS}

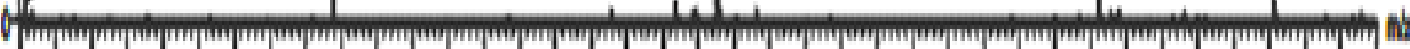
D 臬 MS Spectrum of compound $2 a$ 


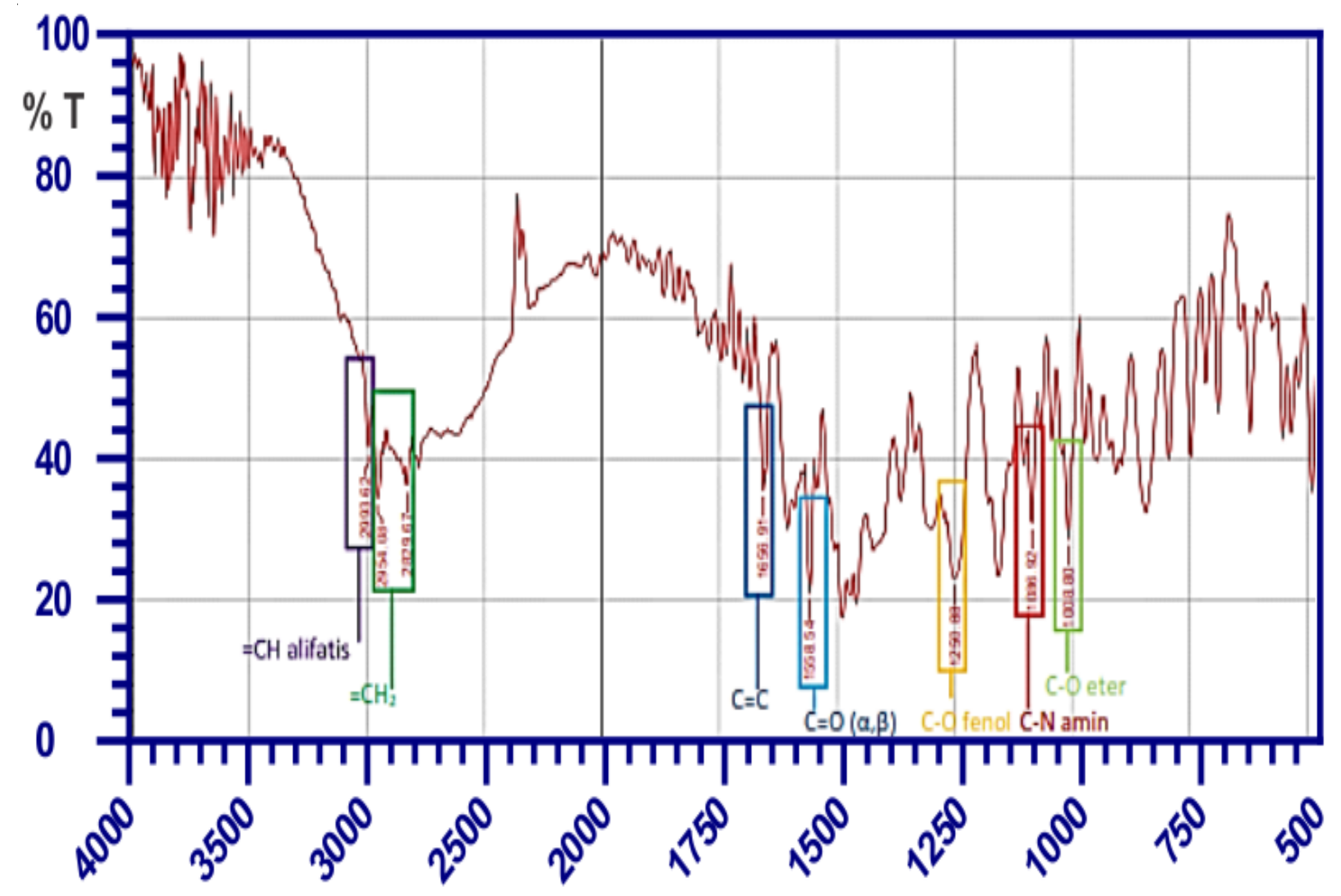

IR Spectrum of compound $2 \mathrm{~b}$ in $\mathrm{KBr}$

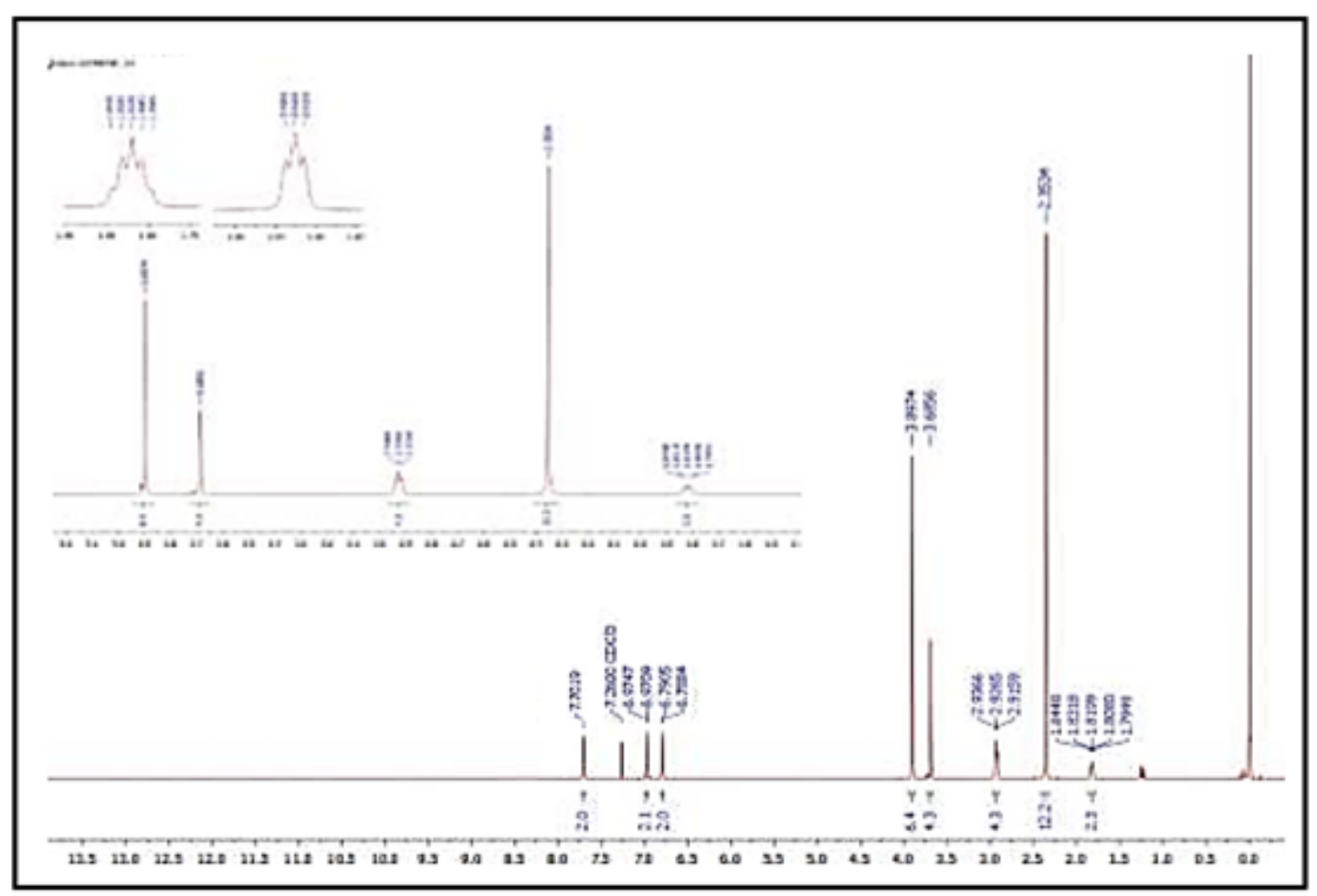

${ }^{1} \mathrm{H}$ NMR Spectrum of compound $2 \mathrm{~b}$ 
Non $>$ socosm 13

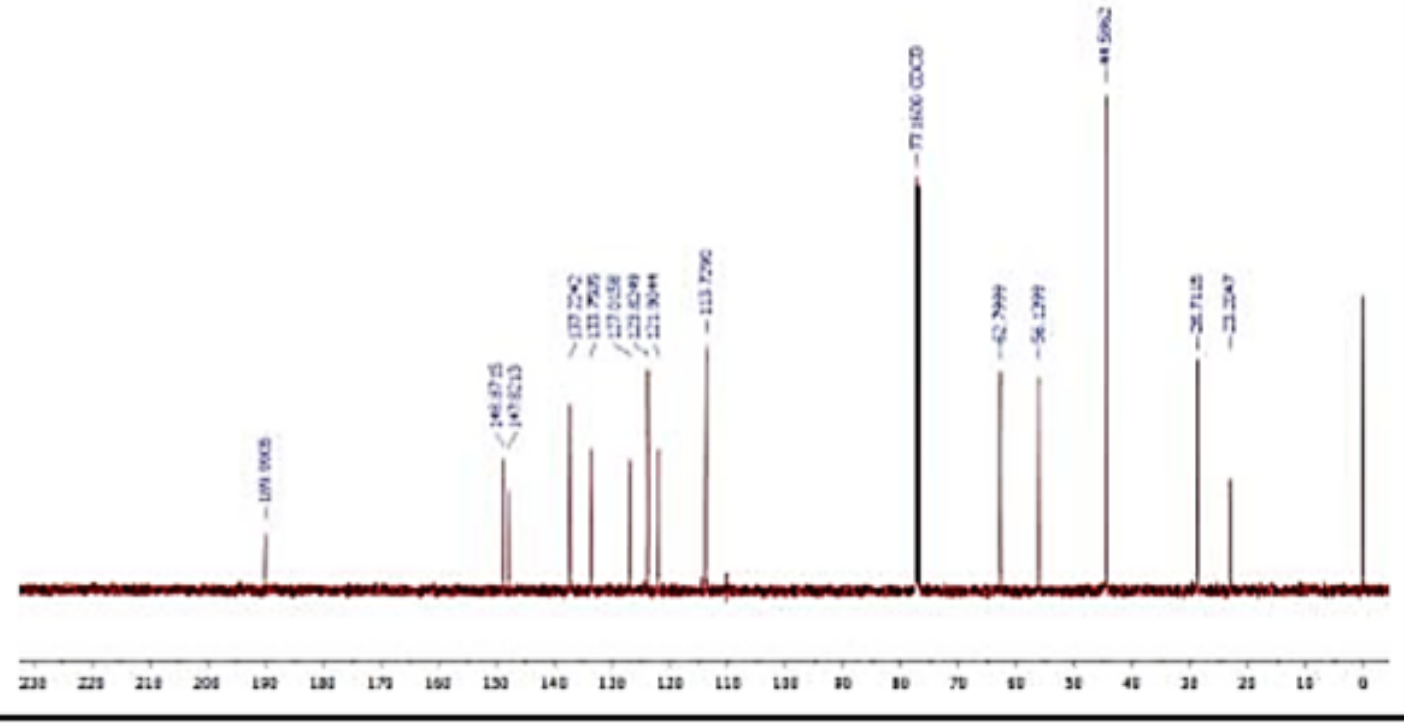

${ }^{13} \mathrm{C}$ NMR Spectrum of compound $2 \mathrm{~b}$

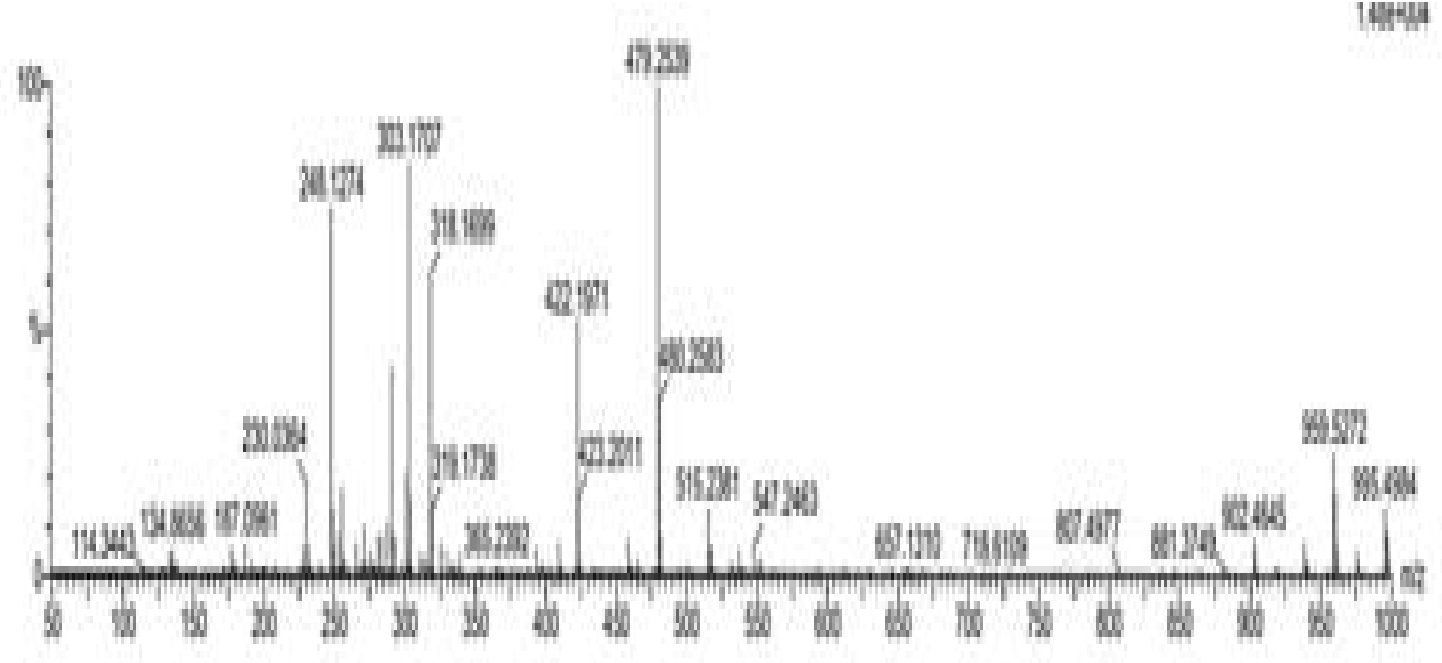

MS Spectrum of compound $2 b$ 

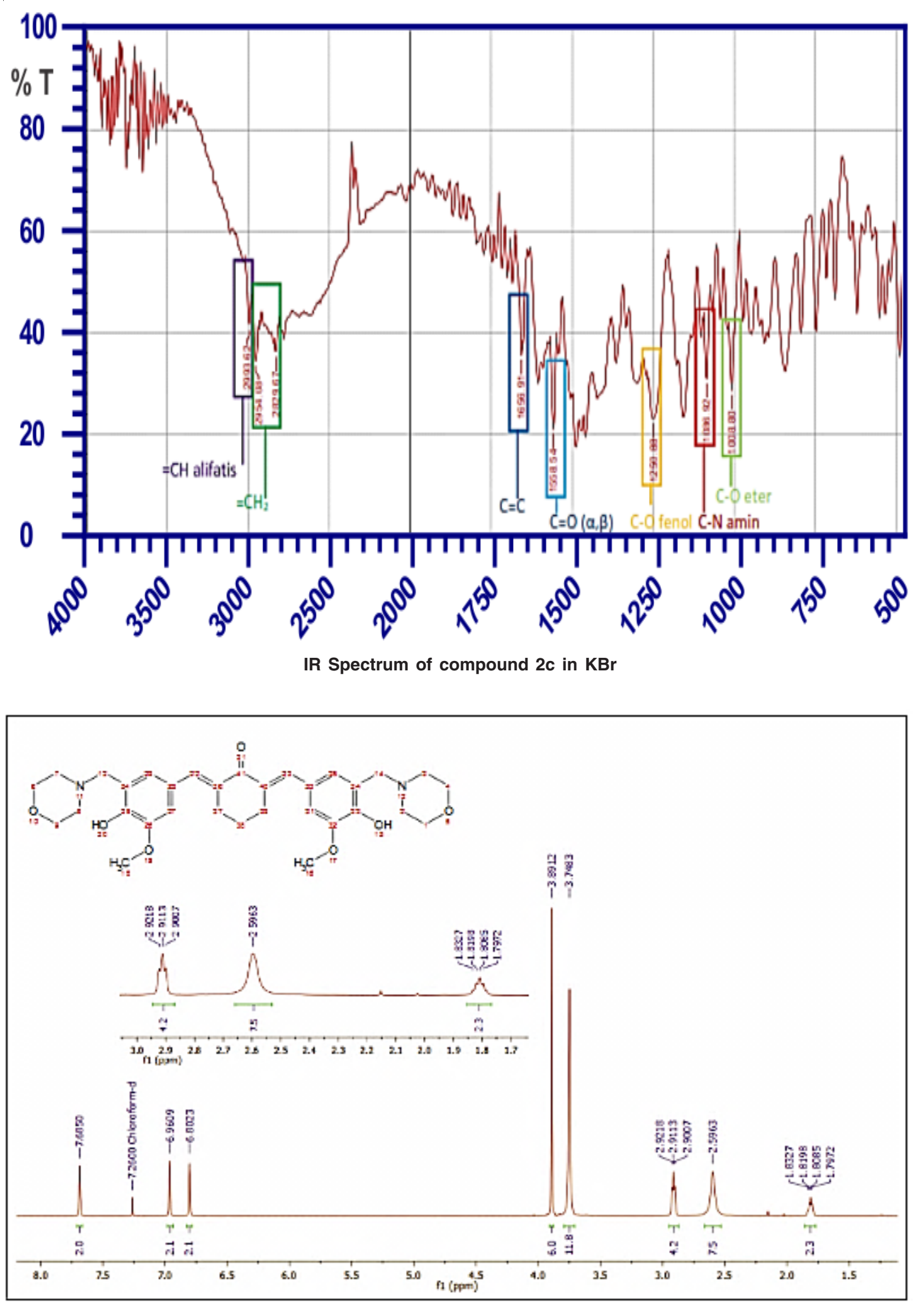

${ }^{1} \mathrm{H}$ NMR Spectrum of compound $2 \mathrm{C}$ 


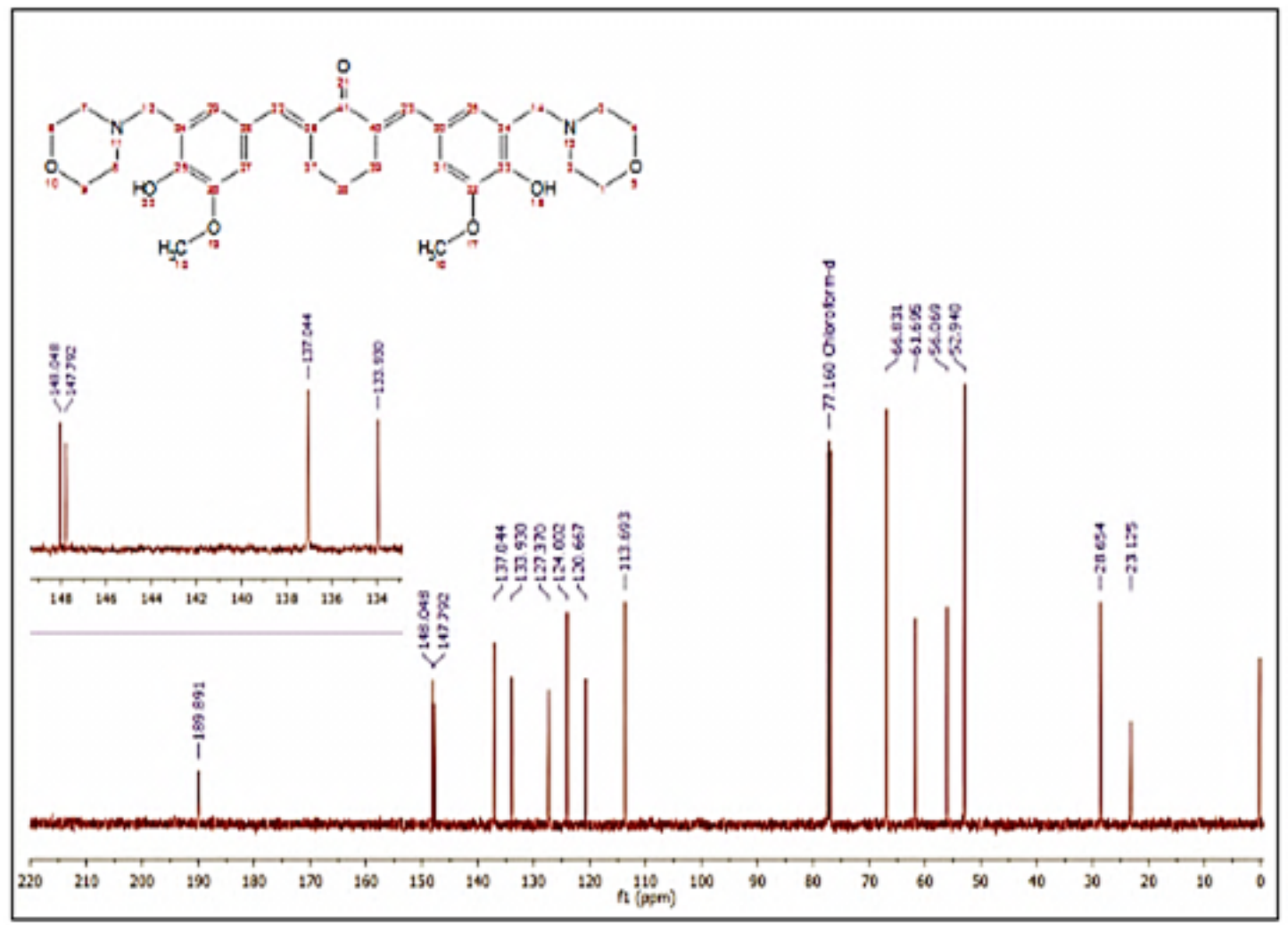

${ }^{13} \mathrm{C}$ NMR Spectrum of compound $2 \mathrm{C}$

Elemental Composition Report

Page 1

Single Mass Analysis

Tolefance $=10.0 \mathrm{mDa} / \mathrm{DBE}: \min =-1.5, \max =50.0$

Element prediction: Off

Monoisolopic Mass, Even Electron lons

3150 formula(e) evaluated with 47 results within limits (up to 5 dosest results for each mass)

Elements Used:

$\begin{array}{llll}C: 0.1000 & \text { H: } 0.1000 \quad \text { N: }: 0.500 \quad 0: 0.500\end{array}$

ESL. TOF

Sand_Seny1_St5s-2914 _oos 5 (0.068) Cm (5)

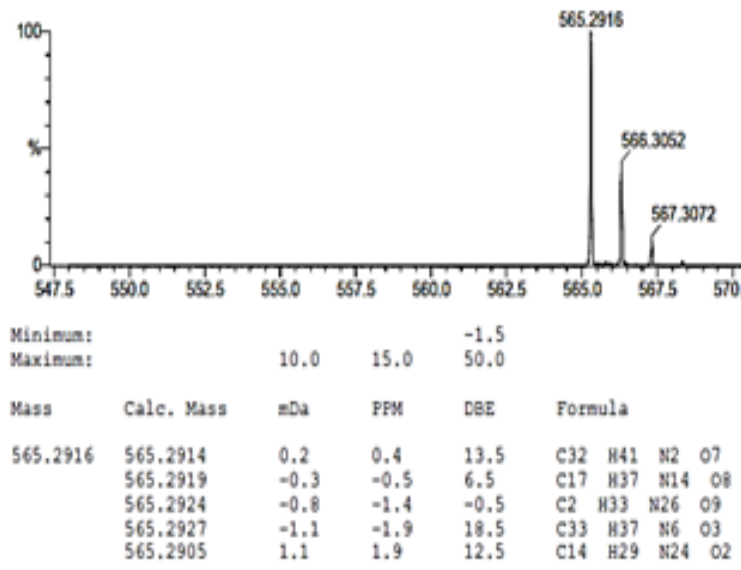




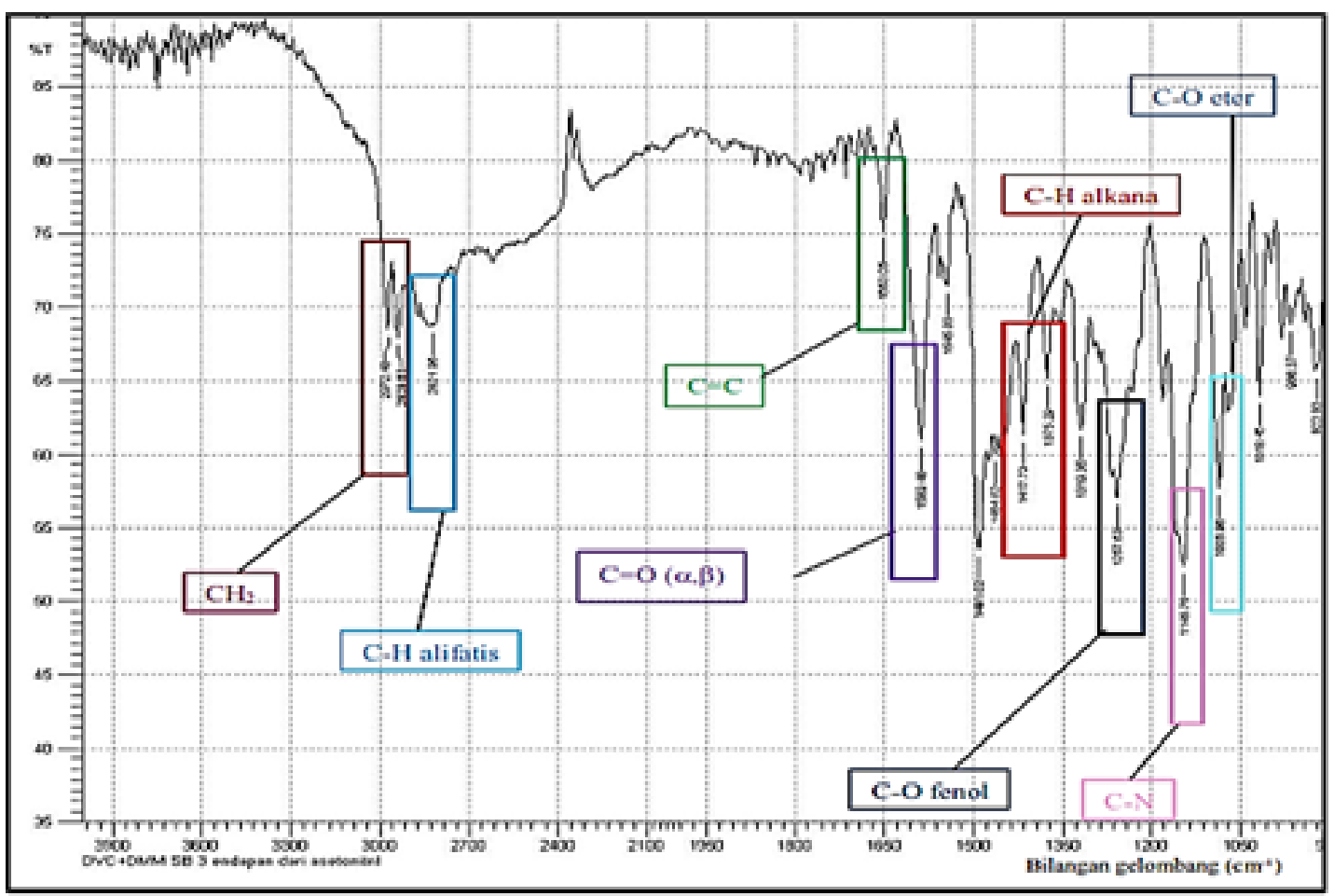

IR Spectrum of compound $2 \mathrm{~d}$ in $\mathrm{KBr}$

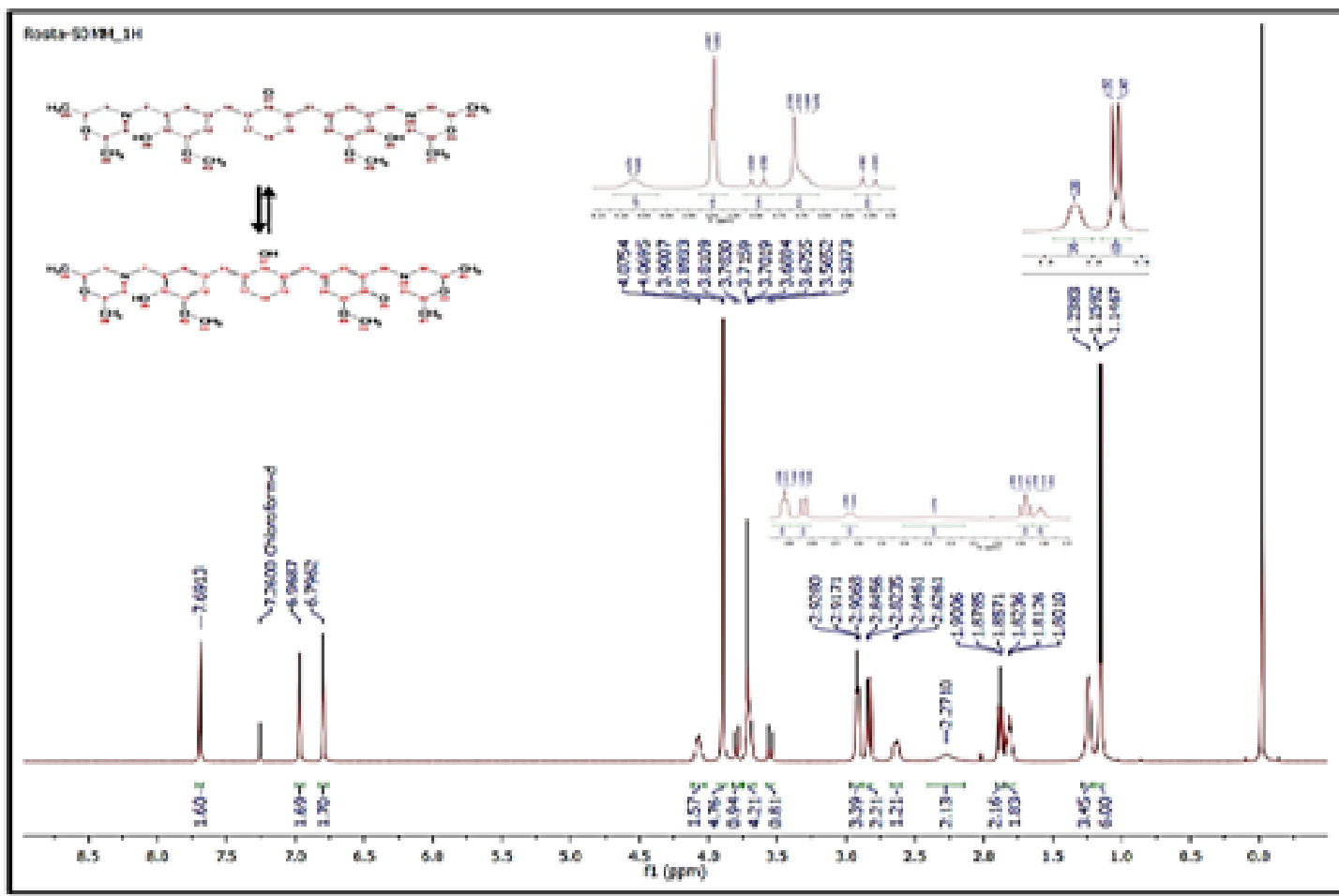

${ }^{1} \mathrm{H}$ NMR Spectrum of compound $2 \mathrm{~d}$ 


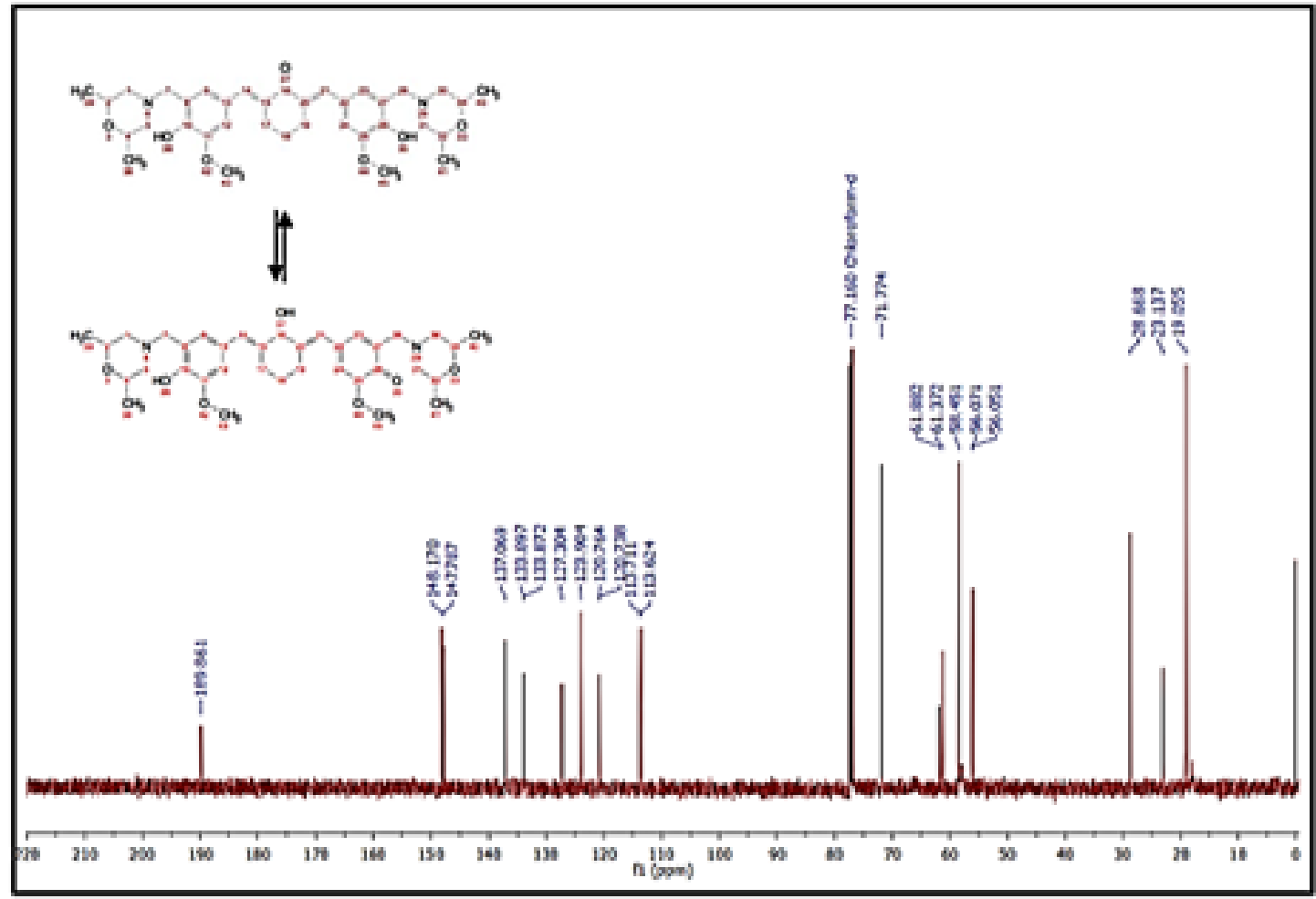

${ }^{13} \mathrm{C}$ NMR Spectrum of compound $2 d$

Elemental Comgovisisn Rosent

Page 1

Singlo Hass Analyis

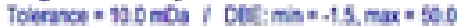

Derest prodston: of

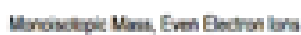

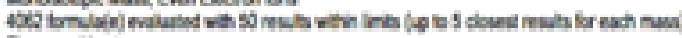

Enerent Uat:

C.0.1000 Hotoco N.0.500 o.0.500

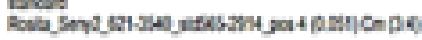

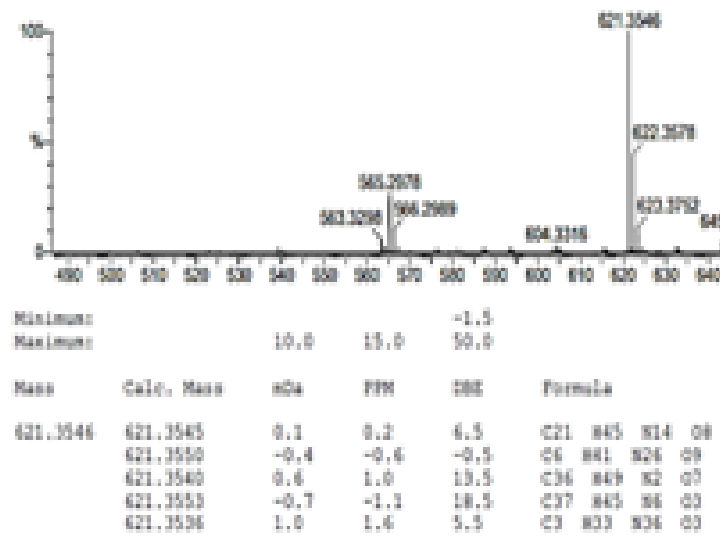



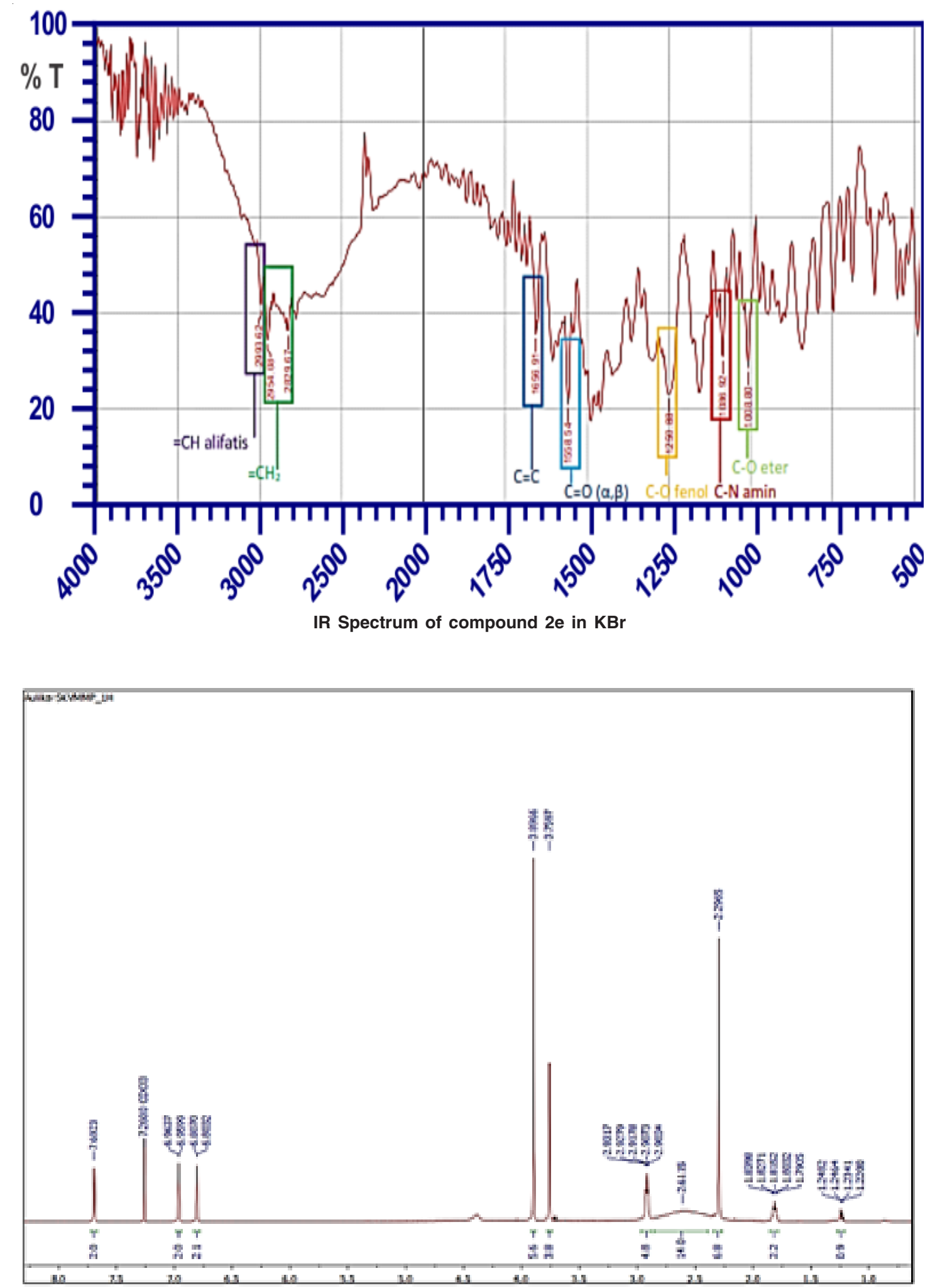

${ }^{1} \mathrm{H}$ NMR Spectrum of compound $2 \mathrm{e}$ 


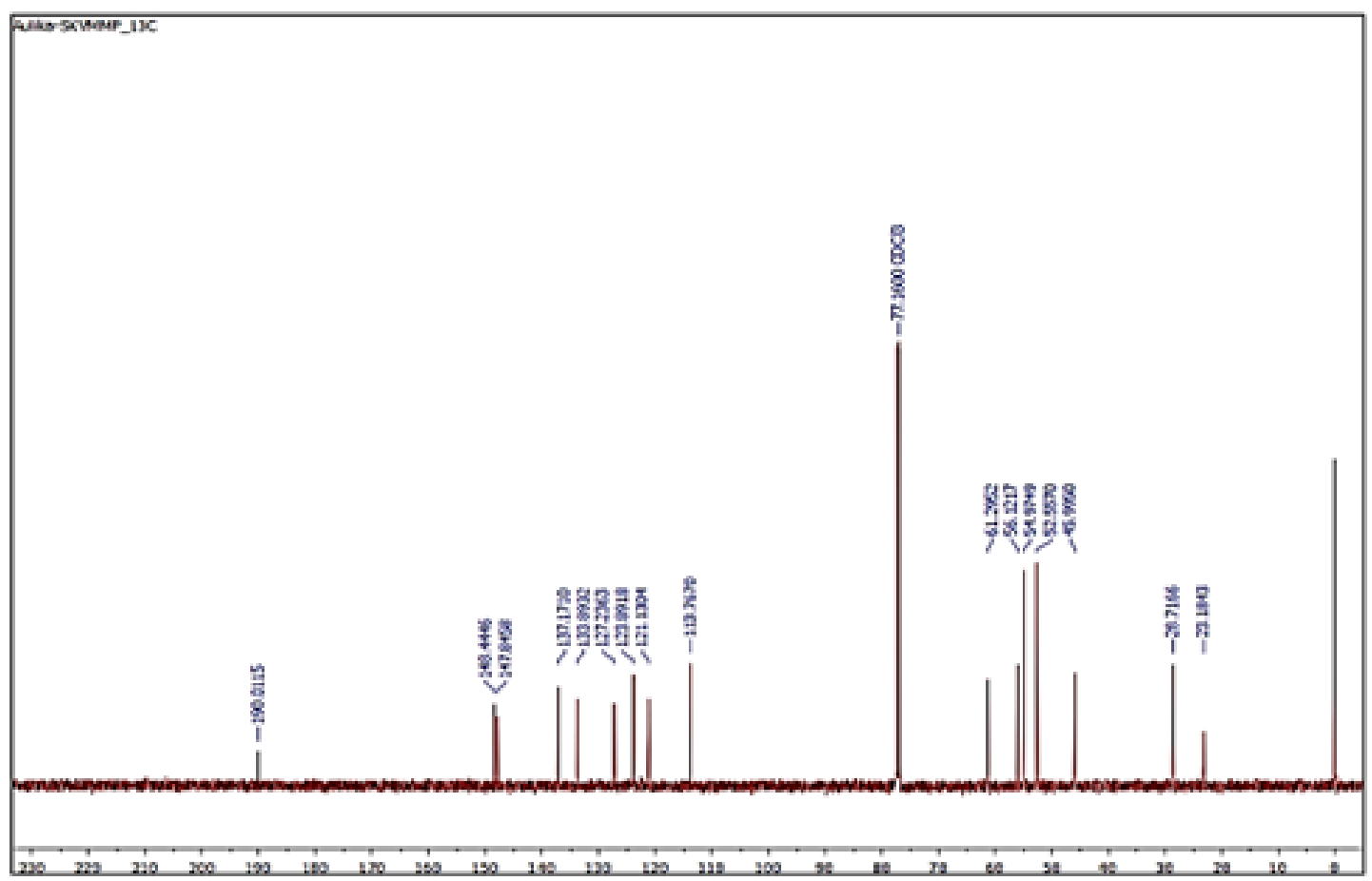

${ }^{13} \mathrm{C}$ NMR Spectrum of compound $2 \mathrm{e}$

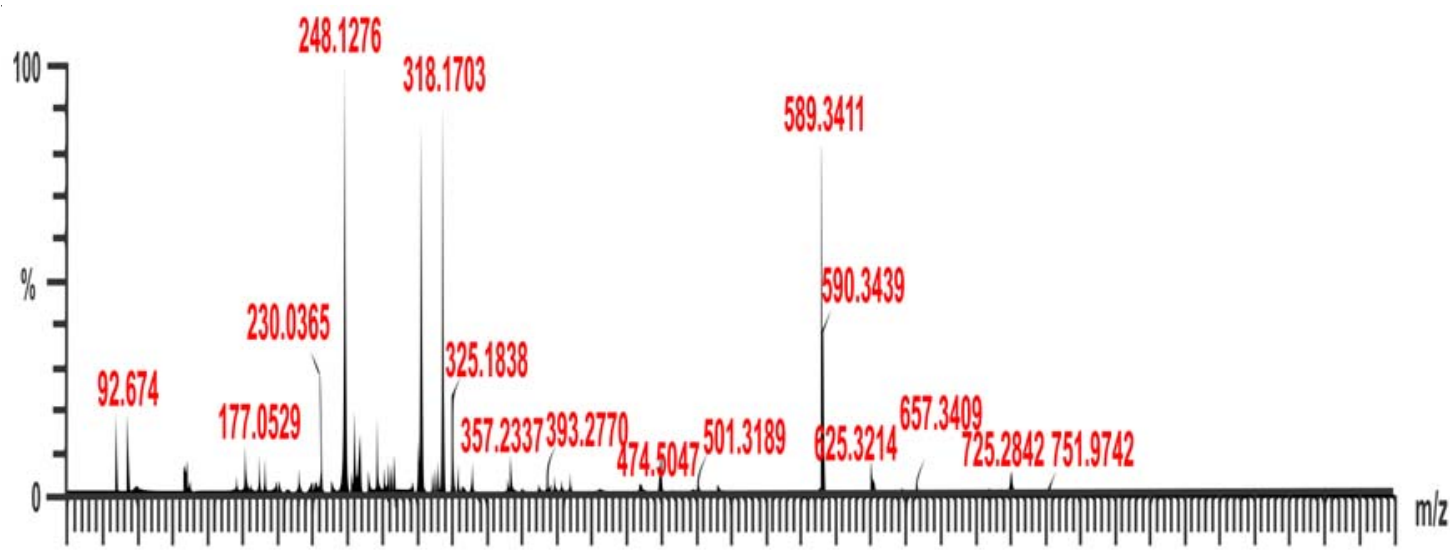

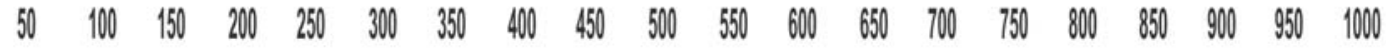

\section{MS Spectrum of compound $2 e$}

(2E,6E)-2,6-bis( $\{[4-h y d r o x y-3-m e t h o x y-5-$ (morpholin-4-ylmethyl)phenyl]methylidene\}) cyclohexan-1-one (2c)

The crude product was purified by recrystallization from ethylacetate-hexane $(1: 1)$ gave a yellow crystalline powder at $63 \%$ yield, $\mathrm{mp}$ $=172-174^{\circ} \mathrm{C} \cdot \mathrm{IR}(\mathrm{KBr}) v_{\max }, \mathrm{cm}^{-1}: 2933,2837,1654$, 1591, 1494, 1305, 1259, 1087, and 1001. ${ }^{1} \mathrm{H}-\mathrm{NMR}$

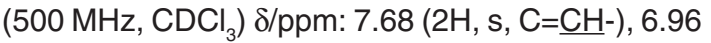

$\left(2 \mathrm{H}, \mathrm{s}, \mathrm{H}_{\mathrm{Ar}}\right), 6.80\left(2 \mathrm{H}, \mathrm{s}, \mathrm{H}_{\mathrm{Ar}}\right), 3.89\left(6 \mathrm{H}, \mathrm{s}, \mathrm{OCH}_{3}\right)$, $3.75\left(12 \mathrm{H}\right.$ of $4 \mathrm{H}, \mathrm{Ar}-\mathrm{CH}_{2}-\mathrm{N}_{\text {morpholine }}$ and $8 \mathrm{H}, \mathrm{C}^{-\mathrm{CH}_{2}}-$ $\left.\mathrm{O}_{\text {morpholine }}\right), 2.91\left(4 \mathrm{H}, \mathrm{t},=\mathrm{C}-\mathrm{CH}_{\underline{2}}-\mathrm{C}_{\text {cyclohexanone }}\right), 2.59$ $\left(8 \mathrm{H}, \mathrm{t}, \mathrm{C}-\mathrm{CH}_{2}-\mathrm{N}_{\text {morpholine }}\right)$, and $1.81\left(2 \mathrm{H}, \mathrm{p}, \mathrm{C}-\mathrm{CH}_{2}-\right.$

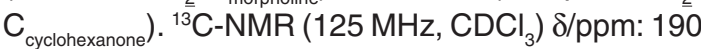
$\left(1 \mathrm{C}, \mathrm{C}=\mathrm{O}_{\text {cyclohexanone }}\right), 148$ and $147\left(4 \mathrm{C}, \mathrm{C}_{\text {Ar-O }}\right), 137$ and 134 (4C, $-\mathrm{C}=\mathrm{C}-), 127,124,121$, and 114 (8C, $\left.\mathrm{C}_{\mathrm{Ar}}\right), 67$ (4C, C-O-C), 62 (2C, Ar-C-N $\left.\mathrm{N}_{\text {morpholine }}\right), 56$ $\left(2 \mathrm{C}, \mathrm{Ar}-\mathrm{O}_{\mathrm{CH}} \mathrm{H}_{3}\right), 53\left(4 \mathrm{C}, \mathrm{C}-\mathrm{N}-\mathrm{C}_{\text {morpholine }}\right), 29$ (2C, =C- 
$\left.\underline{\mathrm{C}}_{2}-\mathrm{C}_{\text {cyclohexanone }}\right)$, and 23 (1C, C- $\left.\underline{\mathrm{C}} \mathrm{H}_{2}-\mathrm{C}_{\text {cyclohexanone }}\right)$. $\operatorname{HRESIMS}(\mathrm{m} / \mathrm{z})$ : found 565.2916 $\left([\mathrm{M}+\mathrm{H}]^{+}\right)$, calculated masses of $\mathrm{C}_{32} \mathrm{H}_{41} \mathrm{~N}_{2} \mathrm{O}_{7}: 565.2914$ (error $0.4 \mathrm{ppm})$.

(2E,6E)-2,6-bis(\{3-[(2,6-dimethylmorpholin-4-yl) methyl]-4-hydroxy-5-methoxyphenyl\} methylidene)cyclohexan-1-one (2d)

The crude product was purified by recrystallization from chloroform-methanol (1:3) gave a pale yellow crystalline powder at $79 \%$ yield, m.p. $=192-194^{\circ} \mathrm{C} . \mathrm{IR}(\mathrm{KBr}), v_{\max }, \mathrm{cm}^{-1}: 2972,2939$, 2821, 1589,1417, 1375, 1653, 1257, 1085 and

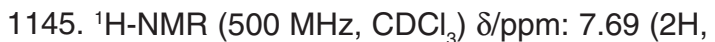
$\mathrm{s}, \mathrm{C}=\mathrm{CH}-), 6.97\left(2 \mathrm{H}, \mathrm{s}, \mathrm{H}_{\mathrm{Ar}}\right), 6.79\left(2 \mathrm{H}, \mathrm{s}, \mathrm{H}_{\mathrm{Ar}}\right), 4,07$ $(2 \mathrm{H}, \mathrm{br}, \mathrm{OH}$ ), 3.89 and 3.90 (two peaks of $6 \mathrm{H}, \mathrm{s}$, $\mathrm{OCH}_{3}$ of the two isomers), $3.71\left(\mathrm{~s}, 4 \mathrm{H}, \mathrm{Ar}-\mathrm{CH}_{2}-\right.$ $\left.\mathrm{N}_{\text {morpholine }}\right), 3,52-3,82\left(4 \mathrm{H}, \mathrm{m}, \mathrm{C}-\mathrm{CH}(-\mathrm{O}-)-\mathrm{C}_{\text {morpholine }}\right)$, $2.92\left(4 \mathrm{H}, \mathrm{t},=\mathrm{C}-\mathrm{CH}_{2}-\mathrm{C}_{\text {cyclohexanone }}\right), 2.83,2.63,2.27$ and 1.87 (four peaks of $8 \mathrm{H}, \mathrm{C}-\mathrm{CH}_{2}-\mathrm{N}$ two isomers), $1.81\left(2 \mathrm{H}, \mathrm{p}, \mathrm{C}-\mathrm{CH}_{2}-\mathrm{C}_{\text {cyclonexanone }}\right), 1.15$ and 1.24 (two peaks of $\mathrm{d}, 12 \mathrm{H}, \mathrm{CH}-\mathrm{CH}_{3}$ of the two isomers). ${ }^{13} \mathrm{C}-\mathrm{NMR}\left(125 \mathrm{MHz}, \mathrm{CDCl}_{3}\right)$ ä/ppm: 190 $\left(1 \mathrm{C}, \mathrm{C}=\mathrm{O}_{\text {cyclohexanone }}\right), 148$ and $147\left(4 \mathrm{C}, \mathrm{C}_{\text {Ar-o }}\right), 137$ and $134(4 \mathrm{C},-\mathrm{C}=\mathrm{C}-), 127,124,121,114\left(8 \mathrm{C}, \mathrm{C}_{\mathrm{Ar}}\right)$, 72 (4C, C-O-C), 62 (2C, Ar-C-N $\left.\mathrm{N}_{\text {mornoline }}\right), 59$ (4C, C$\left.\mathrm{N}-\mathrm{C}_{\text {morpholine }}\right), 56\left(2 \mathrm{C}, \mathrm{Ar}-\mathrm{O}_{\mathrm{CH}}\right), 29\left(2 \mathrm{C},=\mathrm{C}-\mathrm{CH}_{2}-\right.$ $\left.\mathrm{C}_{\text {cyclohexanone }}\right), 23\left(1 \mathrm{C}, \mathrm{C}-\mathrm{CH}_{2}-\mathrm{C}_{\text {cyclohexanone }}\right), 19(4 \mathrm{C}$,
$\left.\mathrm{CH}_{3}-\mathrm{C}_{\text {morpholine }}\right)$. HRESIMS (m/z): found 621.3546 $\left([\mathrm{M}+\mathrm{H}]^{+}\right)$, calculated masses of $\mathrm{C}_{36} \mathrm{H}_{49} \mathrm{~N}_{2} \mathrm{O}_{7}$ : 621.3540 (error 1.0 ppm).

(2E,6E)-2,6-bis(\{4-hydroxy-3-methoxy-5-[(4methylpiperazin-1-yl)methyl]phenyl\} methylidene)cyclohexan-1-one (2e)

The crude product was purified by column chromatography on silica with mixture of chloroform and methanol (1.5:1) as mobile phase gave a brownish orange crystalline powder at $57.0 \%$ yield, m.p. $=163-165^{\circ} \mathrm{C} . \mathrm{IR}(\mathrm{KBr}), v_{\max }, \mathrm{Cm}^{-1}: 2939,2839$, 2797, 1657, 1586, 1500, 1302, 1249, 1086 and 1006. ${ }^{1} \mathrm{H}-\mathrm{NMR}\left(500 \mathrm{MHz}, \mathrm{CDCl}_{3}\right) \delta / \mathrm{ppm}: 7.69(2 \mathrm{H}$, $\mathrm{s}, \mathrm{C}=\mathrm{CH}-), 6.96\left(2 \mathrm{H}, \mathrm{d}, \mathrm{J}=2 \mathrm{~Hz}, \mathrm{H}_{\mathrm{Ar}}\right), 6.80(2 \mathrm{H}, \mathrm{d}$, $\left.\mathrm{J}=2 \mathrm{~Hz}, \mathrm{H}_{\mathrm{Ar}}\right), 4.4(2 \mathrm{H}, \mathrm{br}, \mathrm{OH}), 3.89(6 \mathrm{H}, \mathrm{s}, \mathrm{OCH} 3)$, $3.76\left(4 \mathrm{H}, \mathrm{s}, \mathrm{Ar}-\mathrm{CH}_{2}-\mathrm{N}_{\text {piperazine }}\right), 2.92\left(4 \mathrm{H}, \mathrm{t},=\mathrm{C}-\mathrm{CH}_{2}-\right.$ $\left.\mathrm{C}_{\text {cyclohexanone }}\right), 2.61\left(16 \mathrm{H}, \mathrm{N}-\mathrm{C}-\mathrm{CH}_{\underline{2}}-\mathrm{N}_{\text {piperazine }}\right), 2.29$ $\left(6 \mathrm{H}, \mathrm{s}, \mathrm{CH}_{3}-\mathrm{N}_{\text {piperazine }}\right), 1.81\left(2 \mathrm{H}, \mathrm{p}, \mathrm{C}-\mathrm{CH}_{2}-\right.$

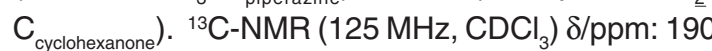
$\left(1 \mathrm{C}, \mathrm{C}=\mathrm{O}_{\text {cyclohexanone }}\right), 148$ and $147\left(4 \mathrm{C}, \mathrm{C}_{\mathrm{Ar}-\mathrm{O}}\right), 137$ and $134(4 \mathrm{C},-\mathrm{C}=\mathrm{C}-) ; 127,124 ; 121$, and $114(8 \mathrm{C}$, $\left.\mathrm{C}_{\mathrm{Ar}}\right), 61\left(2 \mathrm{C}, \mathrm{Ar}-\mathrm{C}-\mathrm{N}_{\text {piperazine }}\right), 56\left(2 \mathrm{C}, \mathrm{Ar}-\mathrm{OCH}_{3}\right), 55$ and $53\left(8 \mathrm{C}, \mathrm{C}-\mathrm{N}-\mathrm{C}_{\text {piperazine }}\right), 46\left(2 \mathrm{C}, \mathrm{CH}_{3}-\mathrm{N}_{\text {piperazine }}\right)$, $29\left(2 \mathrm{C},=\mathrm{C}-\underline{\mathrm{CH}}_{2}-\mathrm{C}_{\text {cyclonexanone }}\right) ; 23\left(1 \mathrm{C}, \mathrm{C}-\underline{\mathrm{C}} \mathrm{H}_{2}-\right.$ $\left.\mathrm{C}_{\text {cyclonexanone }}\right) \cdot \operatorname{HRESIMS}(\mathrm{m} / \mathrm{z})$ : found 589.3411 ([M$\left.\mathrm{H}]^{-}\right)$, calculated masses of $\mathrm{C}_{34} \mathrm{H}_{45} \mathrm{~N}_{4} \mathrm{O}_{5}: 589.3390$ (error $3.6 \mathrm{ppm}$ ).

Table. 1: Free radical-scavenging activity (FRSA) (IC50) of Mannich bases derivatives of Cyclovalone (2a-e)

\begin{tabular}{|c|c|c|c|c|}
\hline No. & $\mathrm{R}-\mathrm{N}-\mathrm{R}$ & Compounds & $\begin{array}{l}\text { IC50 }(\mu \mathrm{M}) 1) \\
(\text { mean } \pm \text { SD })\end{array}$ & Calc. pKa2) \\
\hline 1 & - & 1 & $69.91 \pm 0.37$ & $9.48(\mathrm{ArOH})$ \\
\hline 2 & $\mathrm{C} 2 \mathrm{H} 5-\mathrm{N}-\mathrm{C} 2 \mathrm{H} 5$ & $2 a$ & $39.53 \pm 0.51$ & $8.70(\mathrm{ArOH})$ and $8.69(\mathrm{~N})$ \\
\hline 3 & $\mathrm{CH} 3-\mathrm{N}-\mathrm{CH} 3$ & $2 b$ & $45.83 \pm 0.20$ & $8.70(\mathrm{ArOH})$ and $8.13(\mathrm{~N})$ \\
\hline 4 & & $2 c$ & $163.15 \pm 1.23$ & $8.70(\mathrm{ArOH})$ and $6.27(\mathrm{~N})$ \\
\hline 5 & & $2 d$ & $127.44 \pm 1.06$ & $8.70(\mathrm{ArOH})$ and $6.89(\mathrm{~N})$ \\
\hline 6 & & $2 e$ & $95.96 \pm 0.76$ & $8.70(\mathrm{ArOH}), 7.44(\mathrm{~N} 1)$ and $7.97(\mathrm{~N} 4)$ \\
\hline 7 & - & Quercetin & $21.75 \pm 0.02$ & n.c.3) \\
\hline
\end{tabular}

1) $n=3$; 2) Calculated using MarvinSketch 6.1.0. [16]; 3)n.c. = not calcutated 


\section{Free Radical-Scavenging Activity Evaluation}

The antioxidant activities of Mannich bases of cyclovalone derivatives (2a-e) and cyclovalone (1) were evaluated by the free radicalscavenging activity of stable 2,2-diphenyl-1picrylhydrazyl (DPPH) according to the methodology described by Brand-Williams et al. with a little modification ${ }^{17-18}$. Quercetin was used as reference standard. The test or reference compounds were prepared at five different concentrations in methanol. The test or reference solution $(0.5 \mathrm{~mL})$ was mixed with $0.5 \mathrm{~mL}$ of DPPH radical solution $0.5 \mathrm{mM}$ in methanol and then allowed to stand at room temperature for $30 \mathrm{~min}$. in a dark laboratory condition. The changes in color (from deep violet to light yellow) were measured at $517 \mathrm{~nm}$. The mixture of of methanol $(0.5 \mathrm{~mL})$ and of sample solution $(0.5$ $\mathrm{mL}$ ) serve as a blank. The control solution was prepared by mixing methanol $(0.5 \mathrm{~mL})$ and $\mathrm{DPPH}$ radical solution $(0.5 \mathrm{~mL})$. The experiment for each test compounds was performed in triplicate. The percent free radical-scavenging activity (\% Scavenging) was calculated according to the following equation:

$\%$ Scavenging $=\left[\frac{(\text { A control }- \text { A sample })}{A \text { control }}\right] \times 100$

where A sample is the absorption of DPPH with test or reference compounds and A control is the absorption of DPPH without test compounds. Data obtained was then analyzed using a linear regression equation to determine $\mathrm{IC}_{50}$ of free radical-scavenging activity (FRSA) of the compounds.

\section{RESULTS AND DISCUSSION}

\section{Chemistry}

The title compounds 2a-e were synthesized in two steps by the method summarized in Scheme 1. Vanillin was reacted with cyclohexanone according to the method previously reported to provide cyclovalone (1) $)^{5}$. Treatment of 1 with paraformaldehyde and corresponding secondary amine (a-e) in acetonitrile at reflux temperature for 5-27 $\mathrm{h}$ (TLC monitoring) afforded the title compounds $2 \mathrm{a}-\mathrm{e}$.
The IR spectra of compounds 2a-e appeared $\mathrm{CH}$ aliphatic bands at 2,972-2,830 $\mathrm{cm}^{-1}$ and showed the disappearance of $\mathrm{OH}$ phenolic peak. The bands at 1,246-1,057 $\mathrm{cm}^{-1}$ and 1,000$1081 \mathrm{~cm}^{-1}$ correspond to C-O phenol, C-O ether, and $\mathrm{C}-\mathrm{N}$; while the $\alpha, \beta$-carbonyl groups of the cyclovalone are observed as strong bands at 1,639-1,659 $\mathrm{cm}^{-1}$ and $1589-1591 \mathrm{~cm}^{-1}$. In ${ }^{1} \mathrm{H}-\mathrm{NMR}$ spectra, the protons of two symmetrical aromatic ring remained only four protons appeared at $\delta 6.9$ ppm (2H) and at $\delta 6.8 \mathrm{ppm}(2 \mathrm{H})$ as singlet or doublet with $J=1-2 \mathrm{~Hz}$ indicated that the Mannich base substituted a proton at the ortho position relative to the hydroxyl group of $\mathbf{1}$. The data were supported by the disappearance of $\mathrm{OH}$ phenolic peak in IR spectra caused by intramolecular hydrogen bond formation between the hydroxyl group and $\mathrm{N}$ atom of the Mannich base ${ }^{14-15}$. The structures were further supported by ${ }^{13} \mathrm{C}-\mathrm{NMR}$ and MS spectra of the compounds which showed the complete agreement with the assigned molecular structures.

\section{Antioxidant acitivity}

The antioxidant activities of the synthesized compounds were evaluated using DPPH free radical-scavenger method because of the suitability of the antioxidant mechanism with the compounds. Furthermore, the DPPH analysis is a fast and an uncomplicated test ensuring the reliable result. The mechanism of antioxidant activity briefly is that the phenolic compounds act as the hydrogen donor to reduce the radical molecules, and then the radical antioxidant will be stabilized by electron delocalization in the aromatic system or coupled with other or the same radical antioxidant to give non-radical molecules $3,8,17-19$.

The results of DPPH free radicalscavenging activity of the title compounds are listed in Table 1. All the compounds show free radicalscavenging activity. Compound $2 \mathrm{a}$ is the most potent, with $\mathrm{IC}_{50}=39.0 \mu \mathrm{M}$ or about a half of quercetin's antioxidant activity. The activity of compound $2 \mathrm{a}$ and $2 \mathrm{~b}$ are more potent than that of 1 , while compound $2 \mathrm{e}, 2 \mathrm{~d}$ and $2 \mathrm{c}$ are lower than that of 1 . Structure-activity relationship study of the compounds demonstrates the effect of the alkalinity (pKa) of Mannich substituent of the compound on 
the antioxidant activity (Figure 1). The higher pKa of the Mannich base compound, the higher activity (the lower $\mathrm{IC}_{50}$ ) of the compound. In the previous study was reported that the hydroxyl group of the phenolic antioxidant compound is essential for the activity. The small alkyl substituents (e.g. methyl and ethyl) and electron donating groups (e.g. methoxy) at ortho positions relative to the phenol groups enhanced the activity. On the contrary, bulky alkyl substituents (e.g. isopropyl and t-butyl) retarded the activity ${ }^{5,8}$.

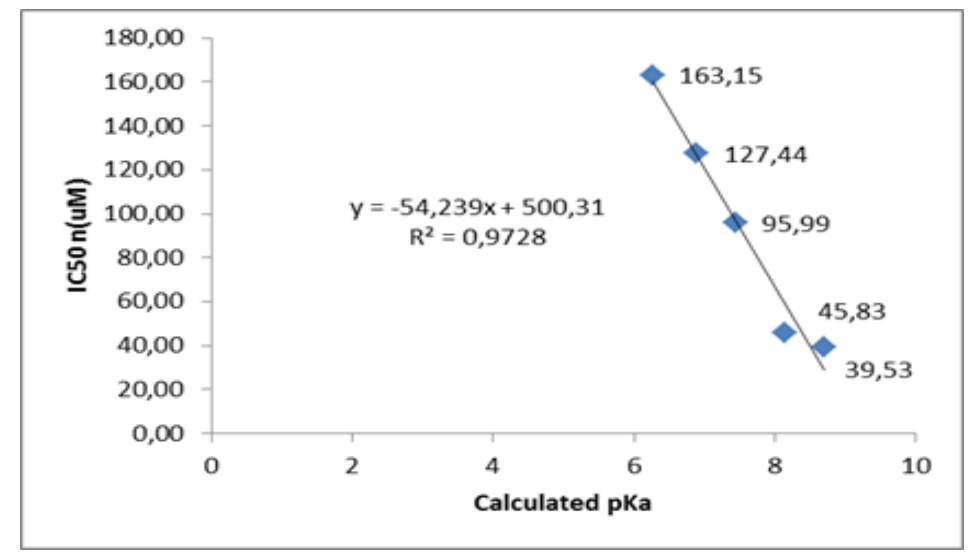

Fig. 1. Relationship between the alkalinity (calculated pKa) and antioxidant activity of the Mannich bases of cyclovalone derivatives

\section{CONCLUSION}

A series of di-Mannich bases of cyclovalone derivatives were synthesized and their free radical-scavenging activity evaluated. The data obtained demonstrated the effect of the basicity of Mannich substituent of the compound on the antioxidant activity. The di-Mannich derivative of cyclovalone with diethylamine and dimethylamine ( $2 a$ and $2 b$ ) exhibited higher free radicalscavenging activity than 1 .

\section{ACKNOWLEDGEMENTS}

The authors thank the Directorate of Research and Community Service Universitas Indonesia, Depok, Indonesia, for the financial support of this research, and the Department of Chemistry, Faculty of Natural Sciences, Bandung Institute of Technology (ITB), Bandung, Indonesia, for recording NMR and HRMS spectral data.

\section{REFERENCES}

1. Young, I. S.; Woodside, J. V. J. Clin. Pathol. 2001, 54, 176-186

2. Roman, G. Eur. J. Med. Chem. 2015, 89, 743-816

3. Dontha, S. Asian J. Pharm. Clin. Res. 2016, 9, Suppl. 2, 14-32.

4. Bayomi, S. M.; El-Kashef, H. A.; El-Ashmawy, M. B.; Nasr, M. N.; El-Sherbeny, M. A.; Badria, F. A.; Abou-zeid, L. A.; Ghaly, M. A.; Abdel-Aziz, N. I. Med. Chem. Res. 2013, 22, 1147-1162

5. Sardjiman, S. S.; Reksohadiprodjol, M. S.; Hakim, L.; van der Goot, H.; Timmerman, $\mathrm{H}$. Eur. J. Med. Chem. 1997, 32, 625-630

6. Nurrochmad, A.; Hakim, A. R.; Margono, S. A.; Sardjiman; Yuniarti, N. Int. J. Pharmacy
Pharm. Sci. 2010, 2 (3), 45-48.

7. Yerdelen, K. O.; Gul, H. I.; Sakagami, H.; Umemura, N. J Enzyme Inhib. Med. Chem., , 2014, Early Online, 1-6.

8. Itokawa, H.; Shi, Q.; Akiyama, T., MorrisNatschke, S. L.; Lee, K. H. Chinese Medicine, 2008, 1-13.

9. Reddy, M. V. B.; Su, C. R.; Chiou, W. F.; Liu, Y. N.; Chen, R.Y.;Bastow, K. F.; Lee, K.H.;Wu, T.S. Bioorg. \& Med. Chem. 2008, 16, 7358-7370.

10. Subramaniapillai, S. G. J. Chem. Sci. 2013, 125 (3), 467-482.

11. Liu, R.; Zhao, B.; Wang, B. E.; Yao, T.; Pang, L.; Tu, Q.; Ahmed, S. M.; Liu, J. J.; Wang, 
J. Molecules, 2012, 17, 14748-14764

12. Bandgar, B.P.;Patil, S. A.; Gacche, R. N.; Korbad, B. L.; Hote, B. S. ; Kinkar, S. N.;Jalde, S. S. Bioorg. Med. Chem. Lett. 2010, 20, 730-733.

13. Bala, S.; Sharma, N.; Kajal, A.; Kamboj, S.; Saini, V. Int. J. Med. Chem. 2014, 1-15.

14. Silverstein, R.M.; Webster, F.X.; Kiemle, D.J. Spectrometric Identification of Organic Compounds, 7th ed., 2005, John Wiley \& Sons, Inc.: New York, NY, USA.
15. Dank, C.; Felsinger, S.; Kirchknopf, B.; Mastalir, M.; Kählig, H.; Roller, A.; Arion, V. B.; Gstach, H. Molecules, 2015, 20, 1686-1711

16. Chemaxon Ltd. http://www.chemaxon.com

17. Brand-Williams W.; Cuvelier M. E.; Berset C. Lebenson Wiss Technol. 1995, 28, 25-30.

18. Kedare, S. B.; Singh. R. P. J. Food Sci. Technol. 2011, 48 (4), 412-422

19. Brewer, M. S. Compr. Rev. Food Sci. Food Safety, 2011,10(4), 221-247 